\title{
Assessing the worldwide epidemiology of sarcoidosis: challenges and future directions
}

\author{
Yvette C. Cozier \\ Affiliation: Slone Epidemiology Center at Boston University, Boston, MA, USA. \\ Correspondence: Yvette C. Cozier, Slone Epidemiology Center at Boston University, 1010 Commonwealth \\ Avenue, Boston, MA 02215, USA. E-mail: yvetteclabu.edu
}

0

@ERSpublications

Population-based studies reveal higher sarcoidosis morbidity and earlier mortality among males http://ow.ly/PcWr304uAKc

Sarcoidosis is a chronic systemic disorder characterised by granulomatous inflammation targeted to an as-yet-unidentified antigen [1]. First described over 100 years ago by Caesar Boeck and others [2], sarcoidosis occurs worldwide and affects men and women of all ages and races [3-5]. The disease shows a consistent predilection for adults, with peak incidence occurring primarily before 40 years of age [3-6], although older age at onset has been widely reported [7-10]. Clinical presentation of sarcoidosis can be quite variable $[11,12]$, ranging from asymptomatic disease [3, 4], or acute onset with resolution [13, 14], while other patients experience chronic progressive illness affecting various organ systems and requiring systemic therapy [13-15]. Pulmonary disease affects the vast majority of diagnosed patients, but other organ systems are often affected as well $[3,4,6]$. Chronic progressive disease may require long-term treatment with corticosteroids, cytotoxics and other agents that have serious side-effects $[3,16,17]$. The lifetime risk of death is estimated to be between $1.0 \%$ and $7.6 \%[17,18]$, with deaths attributed to respiratory, neurological or cardiovascular system involvement $[19,20]$.

While sarcoidosis is described worldwide and in all populations there is considerable disparity in the incidence, prevalence, and disease course between regions and populations. For example, the two groups reported to be most affected by the disease are African Americans and Northern Europeans [3, 21]. It has also been widely reported that sarcoidosis incidence is greater in women than in men [3-5], but the evidence is inconsistent. Data from the USA [22] and Sweden [7] have found similar rates between the two groups, while studies from Poland [23] and Denmark [8] reported higher incidence in men. A recent study in the USA reported higher incidence among women [24], while another study observed a higher prevalence of sarcoidosis for women than men in all racial/ethnic groups [9]. In the USA, sarcoidosis patients experience higher mortality and at younger ages $[25,26]$; age-adjusted mortality rates are greater in females than males, and are consistently higher in black than white patients [25].

Accurate characterisation of sarcoidosis in varied populations is an important step towards formulating strategies for control and treatment of the illness. However, important gaps remain in our knowledge of the epidemiology and aetiology of the disease. These gaps are both highlighted and partially filled by two articles featured in the current issue of the European Respiratory Journal. The first, by ARKEMA et al. [27], estimates incidence and prevalence rates based on data from the Swedish National Patient Register, a comprehensive national database capturing sarcoidosis diagnoses over a 50-year period (1964-2013) among all Swedish residents aged $\geqslant 18$ years. The authors assessed disease frequency in the entire Swedish population by age, sex, education and geography. An important finding from their dataset was that $56 \%$ of prevalent cases and 55\% of incident cases were male, and that the median age at diagnosis for men was nearly a decade younger than that of women (45 versus 54 years). The authors, however, downplay the

Received: Sept 152016 | Accepted: Sept 152016

Conflict of interest: None declared.

Copyright @ERS 2016 
difference in disease frequency between men and women, concluding that it represents "no large difference between the sexes" [27]. While the magnitude of the observed sex differential can be debated, these data suggest that the burden of disease among men may be greater than once understood, and that the sarcoidosis risk profile should be updated to reflect this possibility.

The second study, by Jamilloux et al. [28], is based on 9 years of mortality data from the French Epidemiological Centre for the Medical Causes of Death (CépiDc, INSERM), and represents the largest multiple-cause-of-death analysis in a European country. The authors evaluated sarcoidosis-related mortality compared with the general population, the impact of the most prevalent sarcoidosis-related comorbid conditions, and geographical differences within France. They found that deaths were higher and at younger ages among sarcoidosis patients than among the general population, confirming previous findings from the USA $[25,26]$. Women died less frequently than men, but the average age at death was similar for both sexes. Compared with the general population, sarcoidosis-related death prior to age 65 years was more frequent among males and more frequent among women aged over 65 years. These results, like those of ARKEMA et al. [27], suggest an excess burden of sarcoidosis in a population previously thought to be at lower risk for the disease.

The studies by both ARKema et al. [27] and JAmilloux et al. [28] report geographical variation of sarcoidosis within their respective countries. ARKema et al. [27] report that the highest prevalence of sarcoidosis was observed in the north-western, less densely populated region of the country. Similarly, JAmilloux et al. [28] observed the highest mortality rates in the northern regions of France. Such gradients have been reported previously $[7,8]$ and suggest that environmental and/or occupational exposures may be at play and warrant further systematic study.

Both studies highlight the strengths and limitations of utilising large, population-based databases to study this unique condition. Strengths include the large sample size for each study, which provides representative population estimates of sarcoidosis. The study by ARKEMA et al. [27], in particular, also offers the potential for further in-depth study of important variables as the Swedish dataset has the potential to link data across several national registries. Linkage to medical records data could facilitate phenotyping of cases and their exploration according to sex and geography. Limitations associated with country-specific databases include the inability to directly compare data. As noted, there is considerable variability in the incidence, prevalence and disease course by country and within countries. Specifically, the demographic profile of Sweden varies considerably from that of France, and both differ from other European nations and from the USA. Differences in data sources across countries can also be substantial. Swedish healthcare is universally available and funded by tax payers. Thus, the authors were able to capture nearly $100 \%$ of inpatient hospitalisations, and nearly $90 \%$ of outpatient encounters nationally [27]. Such data are not possible in the USA as healthcare services are not centralised and access to care is not guaranteed to all citizens. Such analyses in the USA are often conducted within healthcare networks $[9,29,30]$ or limited geographic regions $[22,24]$, and may be less representative of the entire US population.

Death certificate and death registry data are imperfect in any country; thus, their use presents limitations. First, the diagnostic accuracy of cases cannot be verified. Further, asymptomatic and mild cases of disease are likely to be underreported, while severe and chronic cases are likely to be overreported. A major limitation of the study by JAmilloux et al. [28] is the lack of data on race or ethnicity, which are not systematically collected. It has been estimated that $15 \%$ of the metropolitan French population is non-white (10\% Northern African, 3.5\% black, 1.5\% Asian) [31]. In addition, an earlier study showed excess disease incidence and morbidity among Afro-Caribbean immigrants to Paris [32]; while in the USA, the highest age-adjusted sarcoidosis-related mortality rates occur among non-Hispanic black patients [25]. Taken together, race and ethnicity are likely to have an impact upon sarcoidosis mortality in France.

Finally, in order to fully appreciate the population morbidity and mortality patterns of sarcoidosis revealed by Arkema et al. [27] and Jamilloux et al. [28], we will need tools that will move us beyond basic descriptive epidemiological analyses. While these large population-based studies are important, their capacity for aetiological research is limited. It is incumbent upon sarcoidosis researchers to look towards alternative datasets and to utilise appropriate epidemiological study designs capable of addressing the aetiology of the disease. These include case-control and prospective (cohort) studies. For example, data obtained from disease registries, or pooled from multiple clinical sites can be used in case-control studies. One such study was the landmark ACCESS (A Case Controlled Etiologic Study of Sarcoidosis) study, a multicentre study sponsored by the National Heart, Lung, and Blood Institute in the USA, which enrolled 736 incident cases of sarcoidosis from 10 clinical centres between November 1997 and May 1999 [33, 34]. Ironically, the ACCESS study was not renewed beyond its initial 5-year funding period as no single risk factor emerged as a dominant influence of disease [35]. Given the possibility that the heterogeneous clinical manifestations of sarcoidosis may be evidence of multiple aetiologies $[11,12]$, it may be time to re-visit this type of study design. 
Additionally, prospective cohort studies can play a critically important role in understanding the aetiology of sarcoidosis. Many such prospective studies exist for the study of other, non-sarcoidosis outcomes (e.g. cancer, cardiovascular disease or asthma), but if properly leveraged, can provide the tools needed to conduct aetiological research into factors such as hormones [36], anthropometry [37] and environment. For example, the Black Women's Health Study (BWHS) [38], a national prospective study of black women in the USA, has followed its 59000 participants for the occurrence of cancer since 1995, and has collected extensive biennial data on demographics, anthropometry, medical and reproductive history, lifestyle factors, medication use, diet, and use of medical care. In 2007, investigators began identifying sarcoidosis cases reported at baseline and over the study follow-up [39]. ARKEMA et al. [27] concluded that, in order to understand the observed disparities by sex, future studies should investigate risk factors by sex separately. The BWHS has taken a first step in this effort. For example, a prospective analysis of reproductive factors found that older age at menopause was associated with a decreased risk of sarcoidosis, suggesting that endogenous oestrogen exposure may be protective among women [36].

In conclusion, there remains much to learn about sarcoidosis. The articles by ArkemA et al. [27] and JAMILloux et al. [28] highlight that sarcoidosis is underappreciated as a cause of morbidity and mortality in European males. They also remind us that we must continue to strategically build upon population-based data in order to enable in-depth, aetiological studies if we wish to move forward from gathering information to preventing disease.

\section{References}

Iannuzzi MC, Rybicki BA, Teirstein AS. Sarcoidosis. N Engl J Med 2007; 357: 2153-2165.

Danbolt N. The historical aspects of sarcoidosis. Postgrad Med J 1958; 34: 245-247.

Bresnitz EA, Strom BL. Epidemiology of sarcoidosis. Epidemiol Rev 1983; 5: 124-156.

Newman LS, Rose CS, Maier LA. Sarcoidosis. N Engl J Med 1997; 336: 1224-1234.

Rybicki BA, Maliarik MJ, Major M, et al. Epidemiology, demographics, and genetics of sarcoidosis. Semin Respir Infect 1998; 13: 166-173.

6 Costabel U, Hunninghake GW. ATS/ERS/WASOG statement on sarcoidosis. Sarcoidosis Statement Committee. American Thoracic Society. European Respiratory Society. World Association for Sarcoidosis and Other Granulomatous Disorders. Eur Respir J 1999; 14: 735-737.

7 Hillerdal G, Nöu E, Osterman K, et al. Sarcoidosis: epidemiology and prognosis. A 15-year European study. Am Rev Respir Dis 1984; 130: 29-32.

8 Byg KE, Milman N, Hansen S. Sarcoidosis in Denmark 1980-1994. A registry-based incidence study comprising 5536 patients. Sarcoidosis Vasc Diffuse Lung Dis 2003; 20: 46-52.

9 Baughman RP, Field S, Costabel U, et al. Sarcoidosis in America. Analysis based on health care use. Ann Am Thorac Soc 2016; 13: 1244-1252.

10 Dumas O, Abramovitz L, Wiley AS, et al. Epidemiology of sarcoidosis in a prospective cohort study of US women. Ann Am Thorac Soc 2016; 13: 67-71.

11 du Bois RM, Goh N, McGrath D, et al. Is there a role for microorganisms in the pathogenesis of sarcoidosis? J Intern Med 2003; 253: 4-17.

12 Prasse A, Katic C, Germann M, et al. Phenotyping sarcoidosis from a pulmonary perspective. Am J Respir Crit Care Med 2008; 177: 330-336.

13 Lofgren S, Lundback H. The bilateral hilar lymphoma syndrome; a study of the relation to age and sex in 212 cases. Acta Med Scand 1952; 142: 259-264.

14 Lofgren S, Lundback $\mathrm{H}$. The bilateral hilar lymphoma syndrome; a study of the relation to tuberculosis and sarcoidosis in 212 cases. Acta Med Scand 1952; 142: 265-273.

15 Johns CJ, Michele TM. The clinical management of sarcoidosis. A 50-year experience at the Johns Hopkins Hospital. Medicine 1999; 78: 65-111.

16 Iannuzzi MC, Fontana JR. Sarcoidosis: clinical presentation, immunopathogenesis, and therapeutics. JAMA 2011; 305: 391-399.

17 Hunninghake GW, Costabel U, Ando M, et al. ATS/ERS/WASOG statement on sarcoidosis. American Thoracic Society/European Respiratory Society/World Association of Sarcoidosis and other Granulomatous Disorders. Sarcoidosis Vasc Diffuse Lung Dis 1999; 16: 149-173.

18 Reich JM. Mortality of intrathoracic sarcoidosis in referral vs population-based settings: influence of stage, ethnicity, and corticosteroid therapy. Chest 2002; 121: 32-39.

19 Perry A, Vuitch F. Causes of death in patients with sarcoidosis. A morphologic study of 38 autopsies with clinicopathologic correlations. Arch Pathol Lab Med 1995; 119: 167-172.

20 Takada K, Ina Y, Noda M, et al. The clinical course and prognosis of patients with severe, moderate or mild sarcoidosis. J Clin Epidemiol 1993; 46: 359-366.

21 Sharma OP. Sarcoidosis around the world. Clin Chest Med 2008; 29: 357-363.

22 Henke CE, Henke G, Elveback LR, et al. The epidemiology of sarcoidosis in Rochester, Minnesota: a population-based study of incidence and survival. Am J Epidemiol 1986; 123: 840-845.

23 Kowalska M, Niewiadomska E, Zejda JE. Epidemiology of sarcoidosis recorded in 2006-2010 in the Silesian voivodeship on the basis of routine medical reporting. Ann Agric Environ Med 2014; 21: 55-58.

24 Ungprasert P, Carmona EM, Utz JP, et al. Epidemiology of sarcoidosis 1946-2013: a population-based study. Mayo Clin Proc 2016; 91: 183-188.

25 Swigris JJ, Olson AL, Huie TJ, et al. Sarcoidosis-related mortality in the United States from 1988 to 2007. Am J Respir Crit Care Med 2011; 183: 1524-1530.

26 Tukey MH, Berman JS, Boggs DA, et al. Mortality among African American women with sarcoidosis: data from the Black Women's Health Study. Sarcoidosis Vasc Diffuse Lung Dis 2013; 30: 128-133. 
Arkema EV, Grunewald J, Kullberg S, et al. Sarcoidosis incidence and prevalence: a nationwide register-based assessment in Sweden. Eur Respir J 2016; 48: 1690-1699.

Jamilloux Y, Maucort-Boulch D, Kerever S, et al. Sarcoidosis-related mortality in France: a multiple-cause-of-death analysis. Eur Respir J 2016; 48: 1700-1709.

29 Rybicki BA, Major M, Popovich J Jr, et al. Racial differences in sarcoidosis incidence: a 5-year study in a health maintenance organization. Am J Epidemiol 1997; 145: 234-241.

30 Birnbaum AD, French DD, Mirsaeidi M, et al. Sarcoidosis in the national veteran population: association of ocular inflammation and mortality. Ophthalmology 2015; 122: 934-938.

31 World Population Review. France Population 2016. http://worldpopulationreview.com/countries/france-population/ Date last accessed: October 12, 2016. Date last updated: August 6, 2016.

32 James DG, Hosoda Y. Epidemiology. In: James DG, ed. Sarcoidosis and Other Granulomatous Disorders. New York, Marcel Dekker, 1994; pp. 729-743.

33 Baughman RP, Teirstein AS, Judson MA, et al. Clinical characteristics of patients in a case control study of sarcoidosis. Am J Respir Crit Care Med 2001; 164: 1885-1889.

34 Design of a case control etiologic study of sarcoidosis (ACCESS). ACCESS Research Group. J Clin Epidemiol 1999; 52: 1173-1186.

35 Rossman MD, Kreider ME. Lesson learned from ACCESS (A Case Controlled Etiologic Study of Sarcoidosis). Proc Am Thorac Soc 2007; 4: 453-456.

36 Cozier YC, Berman JS, Palmer JR, et al. Reproductive and hormonal factors in relation to incidence of sarcoidosis in US Black women: the Black Women's Health Study. Am J Epidemiol 2012; 176: 635-641.

37 Cozier YC, Coogan PF, Govender P, et al. Obesity and weight gain in relation to incidence of sarcoidosis in US black women: data from the Black Women's Health Study. Chest 2015; 147: 1086-1093.

38 Rosenberg L, Palmer JR, Rao RS, et al. Risk factors for coronary heart disease in African American women. Am J Epidemiol 1999; 150: 904-909.

39 Cozier YC, Berman JS, Palmer JR, et al. Sarcoidosis in black women in the United States: data from the Black Women's Health Study. Chest 2011; 139: 144-150. 